\title{
ACM/Springer Mobile Networks and Applications (MONET) Special Issue on "Advanced Mobile Applications and Intelligent Multimedia systems for Ubi-Com"
}

\author{
Jong Hyuk Park • Shu-Ching Chen • Mieso Denko • \\ Sheikh Iqbal Ahamed
}

Published online: 24 March 2010

(C) Springer Science+Business Media, LLC 2010

Ubiquitous Computing (UC) has emerged rapidly as an exciting new paradigm that includes pervasive, grid, and peer-to-peer computing to provide computing and communication services at anytime and anywhere. In order to take the advantages of such services, it is important that intelligent systems be suitable for UC. UC environments have recently drawn enormous attention and interest in both the academic and the industrial research communities as one of the most promising application fields for intelligent services. In fact, the UC domain poses unique challenges such as context fusion, data-mining, multimedia systems and services, communication interoperability, security and safety managements to name just a few.

A total of 29 high-quality papers were received and nine papers were finally selected after rigorous review process by international experts to be published in this issue.

\author{
J. Hyuk Park $(\bowtie)$ \\ Seoul National University of Technology, \\ Seoul, Korea \\ e-mail: jhpark1@snut.ac.kr \\ S.-C. Chen \\ Florida International University, \\ Miami, Fl, USA \\ e-mail: chens@cs.fiu.edu \\ M. Denko \\ University of GUELPH, \\ Guelph, Canada \\ e-mail: denko@cis.uoguelph.ca \\ S. Iqbal Ahamed \\ Marquette University, \\ Marquette, WI, USA \\ e-mail: iq@mscs.mu.edu
}

In the first paper, Naixue Xiong et al. propose the paper entitled "A Fast Formation Flocking Scheme for a Group of Interactive Distributed Mobile Nodes in Autonomous Networks", a novel scheme on the basis of the relative motion theory.

Extensive theoretical analysis and simulation results have demonstrated that the scheme provides the follower nodes an efficient and timely method to follow the leader with the shortest path and the shortest time. In addition, the reported scheme is scalable in the sense that the processing load in each node is not increasing with more nodes in a group.

In the second paper, Kun Yang, Alex Galis, and HsiaoHwa Chen propose a QoS-aware service selection algorithm for pervasive service composition in mobile wireless environment. In this paper, for the first time a QoS model specific for pervasive services is proposed. This model considers not only user-perceived factors but also mobile wireless network characteristics. The formulas to calculate each QoS criterion are also given. This paper has identified some differences between web service composition and pervasive service composition, based on which a local optimal service selection algorithm (LOSSA) is introduced. The corresponding performance results have shown the effectiveness of the proposed algorithms.

The third paper entitled "Tampering in RFID: A Survey on Risks and Defenses" by Gandino, Montrucchio and Rebaudengo deals with the analysis of the data tampering threat in RFID-based information systems. Authors describe the approaches proposed by the research community to protect against data tampering. Also the most important recent studies on privacy and security for RFID-based systems are examined, and the protection given against tampering is evaluated. The paper provides readers with an 
exhaustive overview on risks and defenses against data tampering, highlighting RFID weak spots and open issues.

In the fourth paper entitled "Two-Tier, Scalable and Highly Resilient Key Predistribution Scheme for LocationAware Wireless Sensor Network Deployments" by Unlu and Levi, the authors propose a novel probabilistic key distribution scheme for wireless sensor networks. The assumed deployment model is a zone-based one. However, the zones do not have common keying material. When needed, nodes that belong to the different zones establish secure links via agent nodes, thanks to two-tier network model. According to the simulations, the proposed scheme is highly resilient against node capture attacks and a scalable one. Moreover, it provides good secure connectivity with minimal communication cost.

The fifth paper entitled "A Proxy Mobile IPv6 Based Global Mobility Management Architecture and Protocol" by Huachun Zhou, et al. specifies a Proxy Mobile IPv6 based global mobility management architecture and protocol procedure, named GPMIP. In GPMIP, the mobility management is performed by network entity other than mobile node itself. The benefit is the elimination of the wireless link data delivery tunnel overhead between the mobile node and the access router.

In the sixth paper entitled "An Energy Efficient Middleware Architecture for Processing Spatial Alarms for Mobile Clients", the authors Anand Murugappan and Ling Liu present a middleware architecture for energy efficient processing of spatial alarms for mobile clients, while maintaining low computation and storage costs. Spatial alarms are fundamental techniques for location based advertisement and location based entertainment. The energy-efficient techniques presented in this paper use the concept of safe distance to minimize device wake up times and minimize the number of alarms to be checked on each wake up. Experiments show that the proposed spatial alarms middleware architecture offers significant improvements on battery lifetime of mobile clients while maintaining high accuracy and success rate of spatial alarm services.

The seventh paper "NoiseSPY: A Real-Time Mobile Phone Platform for Urban Noise Monitoring and Mapping" by Eiman Kanjo presents the design, implementation, evaluation, and user experiences of the NoiseSpy application, a sound sensing system that turns the mobile phone into a low-cost data logger for monitoring environmental noise.

The eighth paper entitled "On Prolonging the Lifetime for Wireless Video Sensor Networks" by Ivan Lee, William Shaw, and Jong Hyuk Park presents the study of wireless video sensor network with a mobile sink. The video sensors investigated in this paper is powered by solar energy, with distinct charging and discharging characteristics compares to conventional battery-power video sensors. Communication between the video sensors are structured using an unequal layered clustering topology, which helps improving the network resource allocation for transmitting data in the video sensor network. Performance analysis in terms of network lifetime, tracking error, video distortion of the proposed system is investigated in this paper.

The ninth paper entitled "A Novel Image Mosaicking Technique for Enlarging the Field of View of Images Transmitted over Wireless Image Sensor Networks" by Pazzi, Boukerche, Feng and Huang tackles the problem of high-quality image transmission over wireless sensor networks. To this end, the authors propose innovative image mosaicking and registration techniques that aim at supporting fast delivery of high-quality images. Additionally, image registration accuracy for enlarging field-of-view has been improved and evaluation results show significant reduction in delay, while keeping similar performance and image quality of existing approaches.

Finally, we strongly believe that the selected papers make a significant contribution to researchers, practitioners, and students working in the areas of mobile applications and intelligent multimedia systems.

We would like to express our sincere appreciation to all the authors for their valuable contributions and also to the referees for their cooperation and hard work in reviewing the papers in a timely and professional manner. Our special thanks go to the editorial board for this SI and Prof. Imrich Chlamtac who is the Editor in Chief of this journal, for his supports throughout the whole publication processes. 